0 Journal for ImmunoTherapy of Cancer

\section{Assessing tumor heterogeneity: integrating tissue and circulating tumor DNA (ctDNA) analysis in the era of immuno-oncology - blood TMB is not the same as tissue TMB}

To cite: Fridland S, Choi J, Nam M, et al. Assessing tumor heterogeneity: integrating tissue and circulating tumor DNA (ctDNA) analysis in the era of immuno-oncology - blood TMB is not the same as tissue TMB. Journal for ImmunoTherapy of Cancer 2021;9:e002551. doi:10.1136/jitc-2021-002551

Accepted 12 July 2021
Check for updates

(C) Author(s) (or their employer(s)) 2021. Re-use permitted under CC BY-NC. No commercial re-use. See rights and permissions. Published by BMJ.

${ }^{1}$ Northwestern University Feinberg School of Medicine, Chicago, Illinois, USA

${ }^{2}$ Northwestern University, Evanston, Illinois, USA

${ }^{3}$ Robert H. Lurie Comprehensive Cancer Center of Northwestern University, Northwestern University Feinberg School of Medicine, Chicago, Illinois, USA

Correspondence to

Dr Young Kwang Chae;

young.chae@northwestern.edu

\section{ABSTRACT}

Tissue tumor mutational burden (TTMB) is calculated to aid in cancer treatment selection. High tTMB predicts a favorable response to immunotherapy in patients with non-small cell lung cancer. Blood TMB (bTMB) from circulating tumor DNA is reported to have similar predictive power and has been proposed as an alternative to tTMB. Across many studies not only are tTMB and bTMB not concordant but also as reported previously by our group predict conflicting outcomes. This implies that bTMB is not a substitute for $\mathrm{TTMB}$, but rather a composite index that may encompass tumor heterogeneity. Here, we provide a thorough overview of the predictive power of TMB, discuss the use of tumor heterogeneity alongside TMB to predict treatment response and review several methods of tumor heterogeneity assessment. Furthermore, we propose a hypothetical method of estimating tumor heterogeneity and touch on its clinical implications.

\section{INTRODUCTION}

Tumor mutational burden (TMB) is a clinically approved and frequently used biomarker in the context of immunotherapy. Tissue TMB (tTMB), the total number of mutations per exome, is associated with tumor neoantigen load. ${ }^{1}$ Neoantigens arising from tumor-specific mutations can elicit a robust immune response which is strongly associated with clinical outcome. ${ }^{23}$ A strong correlation between high tTMB and clinical outcomesprogression-free survival (PFS) and overall survival (OS) - has been observed in patients with non-small cell lung cancer (NSCLC) receiving immunotherapy. In contrast, intermediate and low tTMB values correlate with a lower durable clinical response (DCR) rate. ${ }^{4}$

Tissue biopsy, a requirement for measuring $\mathrm{tTMB}$, is invasive and can be impossible to perform in many cases. More importantly, single biopsies do not capture the entire mutational landscape of a tumor, especially when metastases are present. ${ }^{5}$ Some metastases may not even be visible radiographically and thus cannot be evaluated. To compensate for this, clinicians have begun assessing blood TMB (bTMB) from circulating tumor DNA (ctDNA). ${ }^{6}$ Necrotic and apoptotic tumors shed their DNA into circulation. ${ }^{7}$ By monitoring this cell-free circulating DNA (cfDNA), we can less invasively assess the tumor mutational landscape. ${ }^{8}$

Upwards of $94 \%$ of all mutations can be detected in both blood and tissue, with a small percentage of mutations found in one and not the other. ${ }^{9}$ However, bTMB does not yield the same predictions as tTMB. Among patients treated with immune checkpoint inhibitors, high bTMB values were correlated with shorter PFS and $\mathrm{OS}^{6}$. In another work, the DCR rates between bTMB-High and bTMB-Low groups showed no statistically significant difference. ${ }^{10}$ Consequently, tTMB and bTMB do not appear to be equivalent. Furthermore, the small percentage of discordant mutations may carry a predictive power.

Mutations, single nucleotide variations, can be classified as clonal or subclonal. A collection of these mutations can be used to quantify tumor heterogeneity, which has illustrated predictive power in preclinical settings. ${ }^{11}$ In one study, $92 \%$ of tumors that exhibited low heterogeneity (low neoantigen subclonal fraction) and high TMB demonstrated durable clinical benefit with anti-PD-1 (Programmed cell death protein 1) therapy. ${ }^{12}$ Furthermore, high tumor heterogeneity has been observed in patients with colorectal cancer with lower disease-free survival and higher rates of metastases. ${ }^{13}$ A highly heterogeneous breast 
cancer cohort exhibited lower levels of tumor infiltrate, decreased immune responses, and worse outcome. ${ }^{14}$

It has become increasingly clear that there is value in complementing tTMB with other metrics such as bTMB and tumor heterogeneity. Several approaches to measure tumor heterogeneity have been developed and validated. As of this writing, no methods have successfully integrated tTMB, bTMB, and their respective clonality and subclonality characteristics. A thorough analysis of current methods and our own proposed new method may lead to better tumor lineage tracking, earlier detection of resistance-causing mutations, and more effective clinical decision-making. ${ }^{15}$

\section{SINGLE BIOPSY TISSUE-BASED TMB}

Over the relatively short period of time during which immunotherapies have been used, neoantigen burden has become strongly associated with treatment outcome. To estimate this burden, single biopsy tTMB is routinely used. In evaluating a commercial United States Food and Drug Administration (FDA) approved assay for tTMB (Oncomine Tumor Mutational Load assay), Alborelli $e t$ $a t^{4}$ observed a statistically significant relationship between tTMB and outcome metrics. Patients $(\mathrm{n}=76)$ experiencing durable clinical benefit (stable disease for $>6$ months) had a median $\mathrm{tTMB}=8.5$ mut $/ \mathrm{Mb}$, while those with no durable benefit had a median $\mathrm{tTMB}=5$ mut $/ \mathrm{Mb}$ (Dunn's test $\mathrm{p}=0.018$ ). Grouping patients based on a tTMB cut-off of 9 mut/Mb yielded a similar relationship. Patients with a $\mathrm{tTMB} \geq 9$ mut $/ \mathrm{Mb}$ had a median PFS of 16.4 months, while their tTMB $<9$ mut/Mb counterparts had a median PFS of 2.6 months (log-rank Mantel-Cox test $\mathrm{p}=0.0014){ }^{4}$

In another study, tTMB was measured in 1639 patients covering a diversity of cancer indications (FoundationOne Assay). Response rate and PFS were calculated for all patients receiving immunotherapy. Fifty-eight per cent of patients with high tTMB $(>20 \mathrm{mut} / \mathrm{Mb})$ responded positively versus only $22 \%$ of patients with low tTMB responded positively. Furthermore, the median PFS of these two groups was 12.8 versus 3.3 months, respectively, a fourfold difference. For 102 patients within this same cohort who received anti-PD-1/PD-L1 (Programmed death ligand 1) monotherapy, there was a linear correlation between tTMB and response likelihood: $4 \%$ for low (1-5 mut/Mb), 26\% for intermediate (6-19 mut/Mb), $45 \%$ for high (20-50 mut/Mb), and $67 \%$ for very high $(>50 \mathrm{mut} / \mathrm{Mb}) .{ }^{16}$ To bring out further utility from TMB, clinicians have attempted to use it alongside microsatellite instability (MSI).

MSI is determined by measuring the addition or removal of nucleotides from microsatellite tracks. In the context of immunotherapy, MSI has been shown to predict treatment outcome, especially when mismatch repair mutations are considered. ${ }^{17}$ Even though both MSI and TTMB have predictive value, they are not the same. Among 11348 different cancer indications, only $1.5 \%$ were positive for $\mathrm{tTMB}$ and MSI, while specifically in melanoma the rate was $19 \% .{ }^{1}$ When used in conjunction, they are very effective in stratifying patients. Out of 22 patients with MSI high colorectal carcinoma, 13 were tTMB high and $100 \%$ of them responded to immunotherapy. In contrast, $66 \%$ of the patients with low tTMB had progressive disease. More importantly, the median PFS had not been reached for the tTMB high group after 18 months, while the median PFS for the tTMB low group was 2 months. ${ }^{18}$ The relationship between MSI and TMB is of ongoing research interest.

The sequencing methodology used to obtain the raw data is an important consideration. Whole exome sequencing (WES) selects for long stretches of DNA, covering mostly exons, with some introns and intergenic regions. The use of gene panels is an alternative approach that targets a list of genes ranging in number from the low 100s all the way to 1000 . The third and final method is whole genome sequencing (WGS). In this case, all available DNA fragments in the sample are sequenced, capturing interexonic regions. Despite this advantage the high cost and high material requirements of WGS makes it unlikely to be utilized clinically. Most importantly, less costly alternatives like gene panels and WES can be used to effectively measure clinically relevant biomarkers such as TMB and neoantigen load. In comparing the performance of WGS with a small gene panel, TMB high colon cancer tumors showed a 100\% concordance for important tumor driver genes, such as BRAF, KRAS, and NRAS. ${ }^{19}$ Furthermore, tTMB values derived from WES and three commercial gene panels (OCAv3 0.39Mbp, TST170 0.53Mbp, OTML $1.7 \mathrm{Mbp})$ yielded a strong correlation $(0.77,0.84$, and 0.9 respectively; the larger the panel higher the correlation. ${ }^{20}$

\section{MULTIPLE BIOPSY TISSUE-BASED TMB}

Although single biopsies are the standard for most clinicians, they come with a significant number of limitations. Biopsies are invasive and can only be performed on accessible tumors of the appropriate size. Additionally, several studies have shown that a single biopsy does not capture the entire tumor landscape. In evaluating four patients with renal cell carcinoma via multiregion biopsy, only $55 \%$ of all mutations present within the tumor were detected by a single biopsy. Additionally, only $34 \%$ of all mutations were shared by every biopsy, indicating that most mutations cannot be detected by a single biopsy. ${ }^{5}$ This effect has also been observed for tTMB in a study comparing the correlation between single and multiregion tTMB. The authors show that additional biopsies lead to statistically significant fold changes in tTMB, although tTMB stops changing beyond three biopsies. ${ }^{21}$ One biopsy does not capture the mutational state of the tumor. However, an additional two biopsies may be enough.

\section{PLASMA CTDNA AS AN ADDITIONAL BIOPSY}

Liquid biopsy can add another data point to complement situations in which the opportunity for tissue biopsy 
is limited. Blood draws are routine and non-invasive, making them ideal procedures for disease monitoring and treatment assessment. Such applications of liquid biopsy are still in development, and the research has been promising. The presence and abundance of ctDNA has been associated with clinical outcome. In the context of breast cancer, recurrence after stages I and II treatment was exclusively associated with the detection of chromosome 1q23.1 amplification. ${ }^{22}$ Generally, ctDNA levels tend to be associated with tumor burden and may be used to track tumor progression. Pre-surgical and post-surgical ctDNA levels in patients with NSCLC were correlated with outcome. The mutation frequency also declined from $8.88 \%$ to $0.28 \%$, with the largest declines observed in stage I patients. ${ }^{23}$ Beyond total ctDNA levels, tracking bTMB during treatment has also shown clinical value. Wang et al describe the significant response experienced by a patient with metastatic breast cancer to a combination therapy of camrelizumab and vinorelbine. During therapy plasma ctDNA was monitored and bTMB levels declined from 149.2 to 40.88 mut/Mb and the patient experienced 5 months of PFS, prior to which they had not shown response to any other treatment. ${ }^{24}$ In a smaller cohort of six patients with NSCLC a similar trend was observed. After 4 weeks of chemoradiotherapy bTMB decreased significantly as compared with pretreatment levels. ${ }^{25}$ Of four additional patients with NSCLC, only one presented with a significant increase in bTMB, mutation frequency, and overall cell-free DNA levels. This was consistent with disease progression and confirmed radiographically. ${ }^{26}$

Changes in allele frequency of specific mutations in response to treatment may yield significant clinical relevance. An increase in allele frequency was observed for an activating mutation in PIK3CA after paclitaxel, a truncating mutation in $R B 1$ after cisplatin, a truncating mutation in MED1 after tamoxifen and trastuzumab, and a treatment resistance mutation in EGFR after gefitinib. ${ }^{8}$

As with $\mathrm{tTMB}$, the same three sequencing methodologies-gene panel, WES, and WGS-have been applied to ctDNA. WGS has been successfully utilized to study the relationship between PFS/OS and molecular progression (increase in ctDNA)/major molecular response (decrease in ctDNA) ${ }^{27}$ Both WES and gene panels lend equally well to bTMB calculation at the 1021 gene level with Pearson $r$ values ranging from 0.85 to 0.91 depending on indication. ${ }^{21}$

\section{Concordance between bTMB and tTMB}

Given the convenience of ctDNA, bTMB calculation has been readily performed and its predictive value studied. Wang et al observed a strong association between bTMB and PFS in a 34 NSCLC patient cohort treated with immunotherapy. PFS was significantly longer for patients with high bTMB (14.5 months) than their lower bTMB counterparts (5.2 months). However, the more important metric, OS, was not associated with bTMB whatsoever. ${ }^{28}$
Concordance between tTMB and bTMB is of primary research importance. Are bTMB and tTMB the same? Within a 29 NSCLC patient cohort, tTMB and bTMB were correlated: the Pearson $\mathrm{r}=0.47$ for stage I-II patients and 0.58 for stage III-IV patients. Interestingly, single region tTMB and multi region tTMB had a stronger correlation $(\mathrm{r}=0.94)$. This implies that bTMB and tTMB may not convey the same mutational information and most likely are not equivalent. ${ }^{21}$ This observation is further substantiated by a study of 20 patients with NSCLC receiving immune checkpoint inhibitors. Within this cohort, higher bTMB individuals had shorter PFS (45 vs 355 days) and OS (106 days and not reached). ${ }^{29}$ In other works, a high bTMB did not associate with a favorable OS, regardless of the bTMB cut-off that was chosen. ${ }^{30}$

In studying the concordance between tTMB and bTMB it is important to acknowledge the technical differences between tTMB and bTMB assays. Among four commonly used assays FoundationOne $\mathrm{CDx}^{31}$ and Tempus $\mathrm{xT}^{32}$ for tTMB and Guardant $360^{33}$ and GuardantOMNI ${ }^{34}$ for bTMB there is variability between both gene panel size and allele fraction percentage limit of detection (LOD) (figure 1). There appears to be a weak correlation between panel size and limits of detection with an $\mathrm{R}^{2}=0.41$. The bTMB assays generally had a much narrower LOD range then their tTMB counterparts. Individual assay differences in the DNA extraction methods, sequencing library preparation, sequencing parameters, and raw data processing are all sources of bias that may play a significant role in the level of concordance observed between bTMB and tTMB. Furthermore, individual studies and clinical trials choose vastly different tTMB and bTMB cut-offs. One study assessed the performance of an FDA approved tTMB cut-off of $10 \mathrm{mut} / \mathrm{Mb}$ in a metastatic colorectal cohort. They found that a 10 mut/Mb cut-off was not predictive of outcome, while a cut-off of $4.1 \mathrm{mut} / \mathrm{Mb}$ was predictive. For this same cohort the established bTMB cut-off was $28 \mathrm{mut} / \mathrm{Mb}$ illustrating the vast difference between the cut-offs for tissue and blood..$^{35}$ Generally, most research papers studying the relationship between TMB and outcome will select the TMB cut-off that yields the most statistically significant difference between groups.

\section{CLONES, SUBCLONES, AND TUMOR HETEROGENEITY}

A widely accepted and verified explanation for the inequivalence between TTMB and bTMB currently eludes investigators. One possibility is that bTMB may be an index that captures more than just mutational burden. To investigate their previous finding that OS was not associated with bTMB, Wang $e t a l^{28}$ adjusted his bTMB calculation for POPLAR, ${ }^{36}$ an open-label phase 2 randomly controlled advanced NSCLC patient trial, and OAK, ${ }^{37}$ an open-label phase 3 randomly controlled advanced NSCLC patient trial, and a national cancer center cohort. Mutations with an allele frequency $>5 \%$ were classified as high allele frequency (HAF), while the rest as low allele frequency (LAF). HAF-bTMB was strongly correlated 


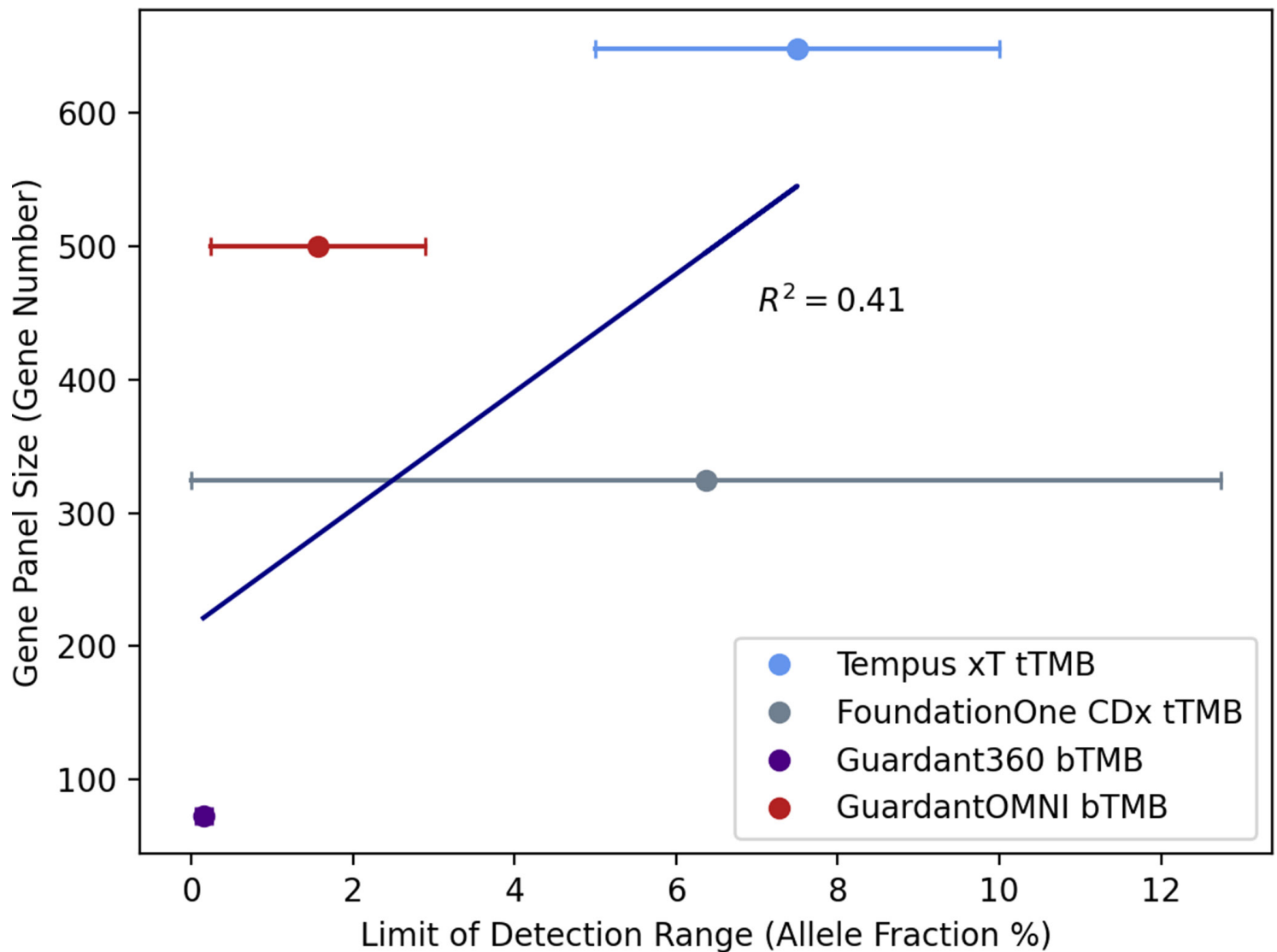

Figure 1 Comparison of gene panel size and limits of detection for four different TMB assays. Each point on the plot represents gene panel size in terms of the number of genes covered by each assay and the mean allele fraction percentage limit of detection. The horizontal lines represent the range of the limit of detection of all alterations. Alterations include single nucleotide variations, Copy Number Variation (CNV), Inserted and/or deleted nucleotides (InDels), and changes in homopolymeric regions. The horizontal lines represent the range of the limit.

with total ctDNA and thus was not associated with positive outcomes. While LAF-bTMB was not associated with total ctDNA. Additionally, LAF-bTMB-High was found to be associated with favorable OS, PFS, and overall response rate $(\mathrm{ORR}) .^{30}$

Liu et $a \rho^{8}$ proposed that BTMB carries two types of bias. The first is called the big tumor burden effect: a larger tumor will shed more cells, resulting in higher amounts of ctDNA. The second is tumor heterogeneity: low frequency mutations represent minor subclones. Mutations present at high and low allele frequencies from larger tumors and minor subclones, respectively, may obscure the true bTMB value. On removing both confounding factors, a new bTMB value was calculated for POPLAR and OAK cohorts. Patients treated with immunotherapy who had a high bTMB exhibited better PFS, OS, and ORR prediction as compared with the Wang et $a t^{28}$ original bTMB calculations. This may imply that the original bTMB values resulted in several bTMB high false positives, which in the clinic would have received immunotherapy to no avail. ${ }^{38}$ While it is clear that patients with a large tumor burden generally have a worse prognosis, patients who exhibit high levels of tumor heterogeneity via their subclonal LAF mutations are currently not stratified in clinical practice.
With more accurate predictive tools, clinicians can more effectively predict treatment outcome by distinguishing between treatment sensitive homogenous cancers and heterogenous cancers capable of harboring treatment resistant subclones. Such an approach could minimize the number of patients receiving treatments from which they are unlikely to benefit.

In preclinical studies, tumor heterogeneity had a significant impact on tumor growth rates regardless of tTMB. At the center of the study are UVB-irradiated melanoma cells, which exhibit higher levels of tTMB than their parental counterparts. These UVB-irradiated cells had on average 2734 more exonic mutations. In mice, cell clones with lower heterogeneity grew at significantly lower rates than both the original parental strain and the UVB-irradiated strain, regardless of tTMB (tTMB ranged from 3600 to 16977 somatic mutations). ${ }^{11}$ These experiments imply that high tumor heterogeneity drives tumor growth and aggressiveness, irrespective of mutational burden. In this experiment, tumor heterogeneity was measured via allele frequency, one of several methods currently utilized in research.

\section{TUMOR HETEROGENEITY METHOD COMPARISON}

Assessing tumor heterogeneity has most commonly been accomplished by quantifying the frequency of different 
Table 1 Tumor heterogeneity methods compared

\begin{tabular}{|c|c|c|c|c|c|}
\hline Method & Summary & Input data type & Output & Publication & Tissue/blood \\
\hline PyClone & $\begin{array}{l}\text { Allele read counts } \\
\text {->cellular prevalence } \\
\text { estimation }\end{array}$ & $\begin{array}{l}\text {.tsv file containing } \\
\text { variant allele } \\
\text { frequency and } \\
\text { copy number } \\
\text { variability }\end{array}$ & Clusters & Roth et $a l^{40}$ & ctDNA and tDNA \\
\hline SciClone & $\begin{array}{l}\text { Like PyClone, but takes } \\
\text { a narrow selection of } \\
\text { genes (copy number } \\
\text { neutral and loss of } \\
\text { heterozygosity free) }\end{array}$ & $\begin{array}{l}\text {.tsv file containing } \\
\text { variant allele } \\
\text { frequency, copy } \\
\text { number variability, } \\
\text { and common LOH } \\
\text { site to exclude }\end{array}$ & Clusters & Miller et $\left.a\right|^{44}$ & tDNA \\
\hline $\mathrm{AFH}$ & VAF/MSAF & VAF and MSAF & AFH Score & Liu et $a l^{47}$ & ctDNA and tDNA \\
\hline MATH & $\begin{array}{l}\text { Width of the VAF } \\
\text { distribution }\end{array}$ & VAF & MATH Score & Mroz and Rocco ${ }^{48}$ & tDNA \\
\hline
\end{tabular}

$\mathrm{AFH}$, allele frequency heterogeneity; ctDNA, circulating tumor DNA; LOH, loss of heterogeneity; MATH, mutant allele tumor heterogeneity; MSAF, maximum somatic allele frequency; tDNA, tumor DNA; VAF, variant allele frequency.

alleles across key genes, their copy numbers, and sequencing depth. The diverse algorithms that have been developed and validated have their respective advantages and disadvantages. A selection of these methodologies are summarized in table 1. Additionally, a computational comparison of some of these methods has been performed by Abécassis et al. ${ }^{39}$

\section{PYCLONE}

PyClone, a Bayesian statistical model, is designed to establish a cluster architecture from ctDNA data. It uses allelic frequencies, read depth, and copy number variation to estimate the size of the cell population that carries a given mutation. If several mutations are present at the same cell population size, then they are considered clustered. Cluster number and size can then be used as an estimation of the number of clones within a sample, in other words, heterogeneity. This approach relies on two assumptions about clonal populations. One, that they are perfect, meaning no single mutation occurs more than once and that for each mutation there is only one somatic genotype. Two, that they are persistent, meaning that mutations do not revert to their somatic genotype. With these limitations in mind, PyClone has been used as a general way of assessing tumor heterogeneity in several studies. ${ }^{40}$

Patients with highly heterogeneous breast cancer ( $>3$ mutation clusters as determined via PyClone) had significantly shorter median PFS (30 weeks) versus their low heterogeneity counterparts (60 weeks). Furthermore, multivariate analysis showed that poor PFS was associated with high heterogeneity $(\mathrm{p}=0.02)$, second only to the type of therapy received (pyrotinib alone vs pyrotinib +chemotherapy) ${ }^{41}$

PyClone has also been applied to understanding the relationship between tumor heterogeneity and neoantigen burden. High neoantigen NSCLC tumors as measured by a bioinformatics pipeline tend to be composed of cells that share a greater number of mutations; in other words, they are more homogenous. These types of tumors were associated with longer PFS. In a second cohort of 64 patients with melanoma, a similar relationship was observed; there was an improved OS in tumors with a low tumor heterogeneity and a high neoantigen burden. ${ }^{12}$

PyClone has been utilized as a primary metric of intratumor heterogeneity in TCGA (The Cancer Genome Atlas) pan-cancer studies. PyClone enabled Morris et al to investigate the clonal landscape of nine different cancer indications, head and neck squamous carcinoma (HNSC), urothelial bladder carcinoma, breast invasive carcinoma (BRCA), kidney renal clear cell carcinoma (KIRC), low-grade glioma (LGG), lung adenocarcinoma, lung squamous cell carcinoma, prostatic adenocarcinoma (PRAD), and skin cutaneous melanoma. They were able to show that for five of the nine indications (HNSC, BRCA, KIRC, LGG, and PRAD) PyClone had a statistically significant relationship with outcome. Most interestingly, immune infiltration was inversely proportional to the 
clone number calculated by PyClone. The authors surmise that the heterogeneity captured by PyClone may be an indicator of a tumor's ability to escape immune surveillance, a factor that must be considered during treatment selection. ${ }^{42}$ Building on this work, Dentro et al compared the performance of PyClone with other clustering-based methodologies. They found that PyClone output relative similarity ranged from 0.5 to 0.81 across 10 methods. When compared with consensus methods PyClone relative similarities ranging from 0.76 to 0.78 , across three methods. Differences in performance are expected due to the many assumptions that each method makes during data processing. Ultimately, it is clear that PyClone has become a mainstay of intratumor heterogeneity measurement studies running from small cohorts all the way to large pan-cancer analyses. ${ }^{43}$

\section{SCICLONE}

A variation of PyClone, SciClone was recently developed to provide higher confidence heterogeneity assessment. This approach takes a narrower range of loci as input. Specifically, these genes come from copy number neutral and loss of heterozygosity free portions of the genome. The intention is to provide a higher confidence measure of variable allele frequency (VAF). VAFs are subsequently clustered using a variational Bayesian mixture model. During validation, SciClone was able to interrogate a cluster architecture in both pre and post treatment samples from Acute Myeloid Leukemia (AML) and breast cohorts. In both samples, it was able to illustrate which clusters responded to treatment and thus were no longer present in post treatment tumors and those which were resistant. Ultimately, SciClone can help clinicians identify targetable subclonal populations. ${ }^{44}$

SciClone has been used as an effective tool to quantify and compare heterogeneity among several biopsies from the same tumor among six patients with colorectal cancer. It was able to identify both shared and distinct subclones between spatially separated areas of each tumor. Although the sample size of this study was small it does provide evidence for the utility of SciClone in tumor heterogeneity research. ${ }^{45}$

The use of SciClone as a method to investigate inflammatory cancers has also been tested. Among a cohort of TCGA breast cancer patients, 20 were inflammatory (IBC) and 23 were non-inflammatory (non-IBC). All the non-IBC patients were found to be made up of at least two clones while $30 \%$ of their IBC counterparts were composed of a single clone. The authors hypothesize that the homogeneity of IBC allowed it to grow more aggressively, while non-IBC clones grew slowly due to inter-clonal competition for resources. Nevertheless, the statistically significant findings of this group show the utility of SciClone. ${ }^{46}$

Lastly, we have begun to see the use of SciClone in clinical case reports. Despite the 'cold' status of ER+/ HER2- metastatic breast cancer Wang et al describe the significant response experience by a patient to a combination therapy of camrelizumab and vinorelbine. During therapy plasma ctDNA was monitored, not only did bTMB levels decline from 149.2 to $40.88 \mathrm{mut} / \mathrm{Mb}$ but also large fluctuations in clonal clusters were observed via SciClone. This suggested to the clinicians that the immunotherapy was effective in targeting specific clones. Unfortunately, the patient did experience progressive disease after 5 months of being progression-free. This study is one of the first to make active use of SciClone as a treatment monitoring tool. ${ }^{24}$

\section{ALLELE FREQUENCY HETEROGENEITY}

Allele frequency heterogeneity (AFH) is a simpler approach which yields a score rather than a set of clusters. It uses the abundance of low frequency alleles as an indicator of high or low heterogeneity. Allele frequency (AF) can be defined as the number of reads for a given mutant at a specified position divided by the total number of reads for that position. Maximum somatic allele frequency (MSAF) represents the allele frequency of the somatic mutant for a given position. The ratio of AF to MSAF can have a wide range of values. A cut-off value must be chosen for this metric to be useful. For example, in prior work a cut-off value of $10 \%$ or 0.1 was chosen. Whether a sample is above or below this cut-off dictates its allele fraction heterogeneity status. Consequently, a sample's heterogeneity can be determined in this binary way. ${ }^{47}$

Although AFH has seen limited use it has been applied to two well studied cohorts. Applying AFH to (POPLAR and $\mathrm{OAK})$, the presence of $\mathrm{AFH}(\mathrm{AF} / \mathrm{MSAF}<10 \%)$ was significantly correlated with unfavorable OS in patients with NSCLC. Median OS was also shorter in AFH present (7.7 months) than in AFH absent (11.7 months) group. More generally, AFH was associated with shorter OS regardless of treatment arms (immunotherapy and chemotherapy). In another independent cohort of 259 patients with NSCLC treated with EGFR Tyrosine Kinase Inhibitors, the presence of AFH was significantly associated with shorter OS $(\mathrm{p}=0.039) .{ }^{47}$

\section{MUTANT ALLELE TUMOR HETEROGENEITY}

Another approach is mutant allele tumor heterogeneity (MATH). The basic unit of this calculation is the mutant allele fraction (MAF), which represents the percentage of sequencing reads that contain a tumor-specific mutant at a given locus. The higher the MAF, the older the mutant is in terms of clonal lineage.

The distribution of all MAF values and their respective abundance can represent the heterogeneity of a tumor sample. For example, MAF values for normal tissue will be either 0.5 or 1 . However, due to the mutated nature of tumor tissue, MAF values can vary significantly from the expected 1 or 0.5 . The extent of this variance is captured by the normalized width of the MAF distribution, otherwise called the MATH score. The higher the score, the 
more the low fraction alleles are present within a tumor sample, thus capturing heterogeneity. ${ }^{48}$

MATH was initially applied to a HNSC cohort of 74 patients. This metric was associated with three poor outcome classes of HNSC: tumors with disruptive TP53 mutations, tumors negative for HPV (Human Papillomavirus), and smokers who were negative for HPV. Although this cohort was small, the relationship between MATH and outcome warrants more investigation. ${ }^{49}$ Extending MATH to a 305 patient TCGA HNSC cohort not only confirmed an association with known prognostic factors (clinical stage, HPV status, TP53 mutation, extent of Copy Number Alterations (CNAs), and mutational load) but also showed that higher MATH scores were independently associated with decreased OS. ${ }^{50}$

Furthermore, the value of MATH as a predictive score was validated in other indications. A seven patient 409 gene panel colon cancer cohort from the UNM Cancer Center Human Tissue Repository showed an association between MATH score and tumor stage. Additionally, stage II individuals who later went on to develop metastasis had higher MATH scores $(\mathrm{p}=0.025)$ than stage II individuals who did not develop metastasis. Per the author's suggestions, stage II individuals with high MATH scores may be ideal candidates for additional treatment and at the very least more aggressive screening. ${ }^{51}$ Another group applied MATH to a TCGA colorectal cancer cohort of 79 patients. As a further confirmation of the work of previous groups, in this cohort higher MATH scores were associated with advanced stage and lymph node metastasis. Post neoadjuvant chemoradiation treatment samples in five of seven patients showed higher MATH scores than their pretreatment counterparts ( 41.7 vs $28.8, \mathrm{p}=0.04){ }^{52}$

MATH was used in studying a breast cancer cohort. MATH scores were dichotomized based on cut-offs that yielded statistically significant differences in survival. High MATH tumors were associated with worse OS, less antitumor CD8 and CD4 T cell infiltration, more immune suppressing regulatory $\mathrm{T}$ cells, and generally lower cytolytic activity. ${ }^{14}$

\section{SHANNON'S DIVERSITY INDEX}

Shannon's Diversity Index (SDI) takes VAFs of a given set of mutated loci and sums the natural log of the probability

Table 2 Scoring matrix

\section{Condition}

Assigned

$\begin{array}{ll}\text { Is this mutation not present in the highest } & 1 \\ \text { frequency cluster as determined by PyClone? } & \\ \text { Is the AF/MSAF ratio for this mutation }<10 \% ? & 1 \\ \text { Is this mutation only detected in ctDNA or } & 1 \\ \text { tissue DNA? } & \end{array}$

AF, allele frequency; ctDNA, circulating tumor DNA; MSAF,

maximum somatic allele frequency. distribution for each across all the loci, yielding a score/ index. A numerical representation of this calculation can be found in table 1. In applying this index to a colorectal cancer cohort, it was observed that stage I-III patients with high SDI exhibited a shorter PFS. In stage IV patients, this association was not observed, most likely because at the metastatic phase other factors play a more significant role. A similar result was obtained for a breast cancer cohort. ${ }^{53}$ High SDI has also been shown to be associated with poor prognostic biomarkers such as prostate specific membrane antigen in prostate cancer. ${ }^{54}$ Another study illustrated a relationship between SDI and adverse pathologic features in breast cancer tumors. These features include high histologic grade, lymphovascular invasion, p53 overexpression, high Ki-67 index, and negative HR. ${ }^{55}$

\section{CALCULATING A SUBCLONALITY SCORE}

As discussed above, mutations found in blood and tissue can differ significantly. Thus, heterogeneity measured via ctDNA and tissue DNA cannot be directly compared. More importantly, we believe that it is the detection and quantification of subclonal mutations that determines how heterogeneous a tumor is. The effectiveness of this approach relies on our ability to determine if a given mutation is subclonal.

We propose a hypothetical index called the Tumor Heterogeneity Index (THI) that we believe may be able to quantify heterogeneity by more constantly differentiating between clonal and subclonal mutations. First, we must score each mutation. In our initial iteration we focus on three mutation conditions (table 2). Although we ascribe one point to each condition, during future validation studies we expect that to optimize this approach the weight of each condition will need to be carefully considered.

Once all mutated loci have been scored, we then calculate the fraction of all alleles that have a score greater than or equal to 2. We call this ratio the THI. By performing this calculation for both tissue and blood samples, we obtain tissue THI (tTHI) (figure 2B) and blood THI (bTHI) (figure 2A), respectively. The mean of these two values we call the composite THI (cTHI). ${ }^{56}$

Subclonality scores and indexes like cTHI may act as another tool in effectively predicting outcome. In addition to being used on its own, this metric can be coupled with others. Much the same as allele frequency cut-offs were used by Liu et $a l^{38}$ subclonality can be used alongside TMB to minimize bias. Although this approach needs to be validated, it does open the door for further investigation.

\section{CONCLUSION}

Even though TMB is actively used in clinical decisionmaking, evidence across multiple studies shows that TMB derived from tissue versus blood can have varying predictive value. Additionally, biopsies obtained from different 


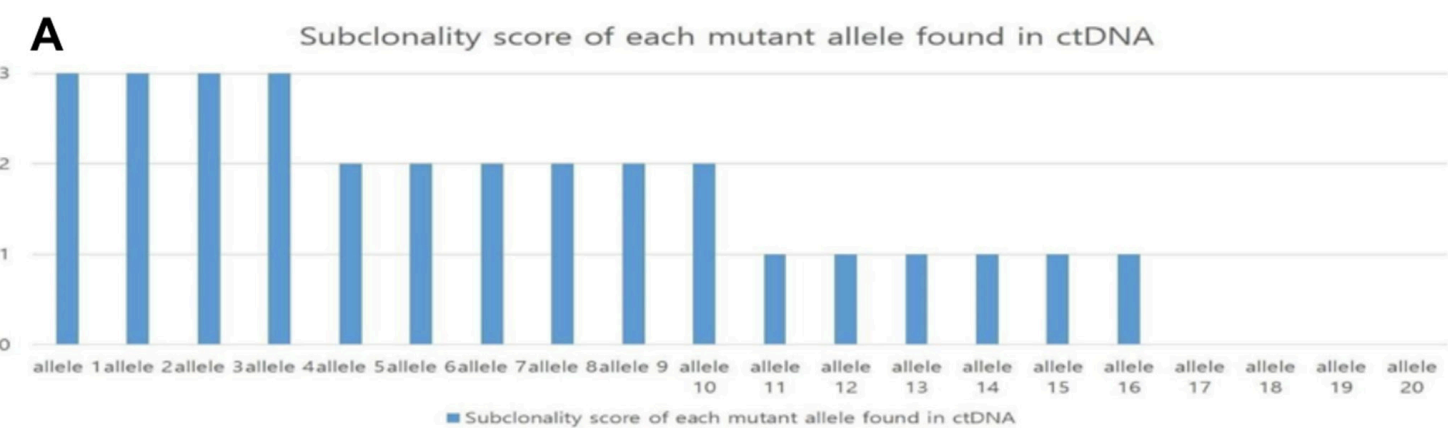

B Subclonality score of each mutant allele found in tissue DNA

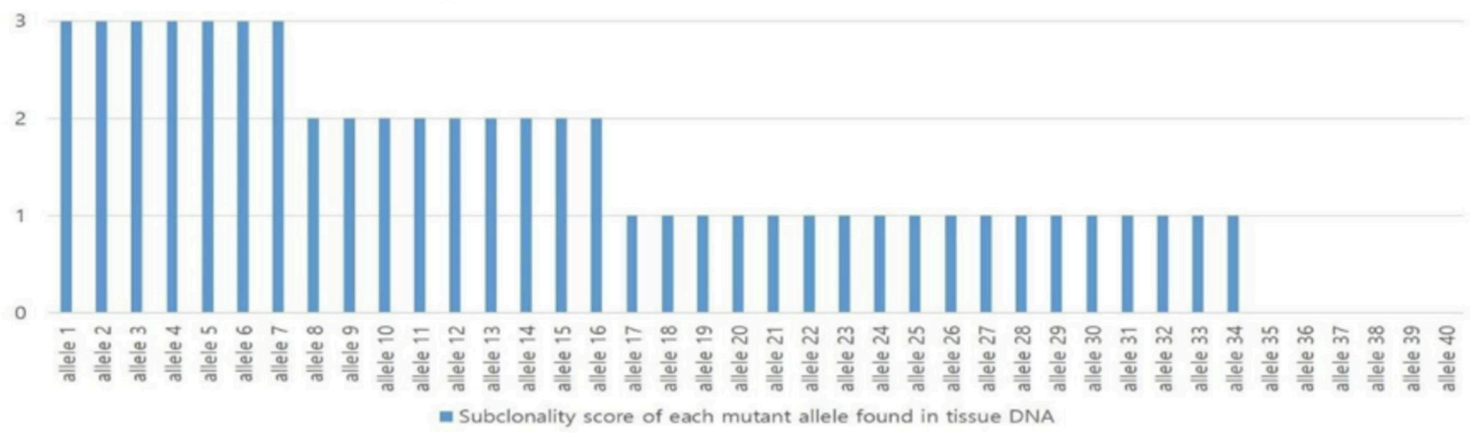

Figure 2 Hypothetical distribution of alleles. (A) Hypothetical distribution of all alleles found in ctDNA, blood Tumor Heterogeneity Index $(\mathrm{bTHI})=$ the number of alleles with a subclonality score greater than or equal to $2 /$ the total number of alleles found in ctDNA=10/20=50\%. (B) Hypothetical distribution of all alleles found in tissue DNA, tissue Tumor Heterogeneity Index $(t T H I)=$ the number of alleles with a subclonality score greater than or equal to $2 /$ the total number of alleles found in tissue DNA $=16 / 40=40 \%$. Thus, the composite THI for these two samples is $45 \%$. ctDNA, circulating tumor DNA.

parts of the same tumor are inherently variable. The same is true for blood samples obtained before, during, and after treatment. All these observations make it difficult to accurately capture the mutational landscape of a tumor within a given patient. However, considering both tumor and blood biopsy and generating a score based on the presence and frequency of certain mutations, we believe that a more accurate index can be calculated. The cTHI is one example of such an index, which can be used on its own and in conjunction with other metrics. The next step is to test and validate the value of cTHI on both publicly and privately available data sets. This is crucial for the field of precision medicine to move forward and become a more accurate and effective means of improving treatment outcomes.

Acknowledgements We would like to thank all the authors involved and Northwestern University for providing access to necessary journal and reference materials.

Contributors Conception and design: SF, JC, MN, YKC; Administrative support: All authors; Provision of study materials: SF, JC, MN, YKC; Manuscript writing: SF; Manuscript editing: All authors; Final approval of manuscript: All authors.

Funding The authors have not declared a specific grant for this research from any funding agency in the public, commercial or not-for-profit sectors.

Competing interests YKC would like to disclose research grants from AbbVie, BMS, Biodesix, Lexent Bio, and Freenome. He also has/had honoraria/advisory board roles for Roche/Genentech, BMS, AstraZeneca, Merck, Foundation Medicine, Counsyl, Neogenomics, Guardant Health, Boehringer Ingelheim,
Biodesix, Immuneoncia, Lilly Oncology, Merck, Takeda, Pfizer, Tempus, Lunit, Jazz Pharmaceuticals. All other authors have nothing to disclose.

Patient consent for publication Not required.

Provenance and peer review Not commissioned; externally peer reviewed.

Open access This is an open access article distributed in accordance with the Creative Commons Attribution Non Commercial (CC BY-NC 4.0) license, which permits others to distribute, remix, adapt, build upon this work non-commercially, and license their derivative works on different terms, provided the original work is properly cited, appropriate credit is given, any changes made indicated, and the use is non-commercial. See http://creativecommons.org/licenses/by-nc/4.0/.

ORCID iD

Stanislav Fridland http://orcid.org/0000-0001-7200-7154

\section{REFERENCES}

1 Vanderwalde A, Spetzler D, Xiao N, et al. Microsatellite instability status determined by next-generation sequencing and compared with PD-L1 and tumor mutational burden in 11,348 patients. Cancer Med 2018;7:746-56.

2 Schumacher TN, Schreiber RD. Neoantigens in cancer immunotherapy. Science 2015;348:69-74.

3 Thorsson V, Gibbs DL, Brown SD, et al. The immune landscape of cancer. Immunity 2018;48:e14:812-30.

4 Alborelli I, Leonards K, Rothschild SI, et al. Tumor mutational burden assessed by targeted NGS predicts clinical benefit from immune checkpoint inhibitors in non-small cell lung cancer. $J$ Pathol 2020;250:ii59.

5 Gerlinger M, Rowan AJ, Horswell S, et al. Intratumor heterogeneity and branched evolution revealed by multiregion sequencing. $N$ Engl J Med 2012:366:883-92.

6 Davis AA, Chae YK, Agte S, et al. Comparison of tumor mutational burden (TMB) across tumor tissue and circulating tumor DNA (ctDNA). JCO 2017;35:e23028. 
7 Jahr S, Hentze H, Englisch S, et al. Dna fragments in the blood plasma of cancer patients: quantitations and evidence for their origin from apoptotic and necrotic cells. Cancer Res 2001;61:1659-65.

8 Murtaza M, Dawson SJ, Tsui DW. Non-Invasive analysis of acquired resistance to cancer therapy by sequencing of plasma DNA. Annals of Oncology 2014;25:iv36.

9 Chae YK, Davis AA, Carneiro BA, et al. Concordance between genomic alterations assessed by next-generation sequencing in tumor tissue or circulating cell-free DNA. Oncotarget 2016;7:65364-73.

10 Fang W, Ma Y, Yin JC, et al. Combinatorial assessment of ctDNA release and mutational burden predicts anti-PD(L)1 therapy outcome in nonsmall-cell lung cancer. Clin Trans/ Med 2020;10:331-6.

11 Wolf $\mathrm{Y}$, Bartok O, Patkar S, et al. Uvb-Induced tumor heterogeneity diminishes immune response in melanoma. Cell 2019;179:219-235. e21.

12 McGranahan N, Furness AJS, Rosenthal R, et al. Clonal neoantigens elicit $\mathrm{T}$ cell immunoreactivity and sensitivity to immune checkpoint blockade. Science 2016;351:1463-9.

13 Joung J-G, Oh BY, Hong HK, et al. Tumor heterogeneity predicts metastatic potential in colorectal cancer. Clin Cancer Res 2017;23:7209-16.

14 McDonald K-A, Kawaguchi T, Qi Q, et al. Tumor heterogeneity correlates with less immune response and worse survival in breast cancer patients. Ann Surg Oncol 2019;26:2191-9.

15 Swanton C. Intratumor heterogeneity: evolution through space and time. Cancer Res 2012;72:4875-82.

16 Goodman AM, Kato S, Bazhenova L, et al. Tumor mutational burden as an independent predictor of response to immunotherapy in diverse cancers. Mol Cancer Ther 2017;16:2598-608.

17 Sahin IH, Akce M, Alese O, et al. Immune checkpoint inhibitors for the treatment of MSI-H/MMR-D colorectal cancer and a perspective on resistance mechanisms. Br J Cancer 2019;121:809-18.

18 Schrock AB, Ouyang C, Sandhu J, et al. Tumor mutational burden is predictive of response to immune checkpoint inhibitors in MSI-high metastatic colorectal cancer. Ann Oncol 2019;30:1096-103.

19 Echejoh G, Liu Y, Chung-Faye G, et al. Validity of whole genomes sequencing results in neoplasms in precision medicine. J Clin Pathol : 2020. doi:10.1136/jclinpath-2020-206998. [Epub ahead of print: 29 Oct 2020].

20 Endris V, Buchhalter I, Allgäuer M, et al. Measurement of tumor mutational burden (TMB) in routine molecular diagnostics: in silico and real-life analysis of three larger gene panels. Int $J$ Cancer 2019;372.

21 Zhang Y, Fang W, Yang Y, et al. P102 the correlations of tumor mutational burden among Single-region tissue, Multi-region tissues and blood in NSCLC. J Thorac Oncol 2018:13:S1085.

22 Goh JY, Feng M, Wang W, et al. Chromosome 1q21.3 amplification is a trackable biomarker and actionable target for breast cancer recurrence. Nat Med 2017:23:1319-30.

23 Guo N, Lou F, Ma Y, et al. Circulating tumor DNA detection in lung cancer patients before and after surgery. Sci Rep 2016;6:33519.

24 Wang R, Yang Y, Ye W-W, et al. Case report: significant response to immune checkpoint inhibitor camrelizumab in a heavily pretreated advanced ER+/HER2- breast cancer patient with high tumor mutational burden. Front Oncol 2021:10.

25 Yang X, Zhu Z. Blood-based TMB detection and dynamic monitor in local advanced non-small cell lung cancer (NSCLC) patients. JCO 2019;37:e20039.

26 Friedlaender A, Nouspikel T, Christinat Y, et al. Tissue-plasma TMB comparison and plasma TMB monitoring in patients with metastatic non-small cell lung cancer receiving immune checkpoint inhibitors. Front Oncol 2020;10:142.

27 Davis AA, lams WT, Chan D. Early assessment of molecular progression and response by whole-genome circulating tumor DNA in advanced solid tumors. Mol Cancer Ther. 2019.

28 Wang Z, Duan J, Cai S, et al. Assessment of blood tumor mutational burden as a potential biomarker for immunotherapy in patients with non-small cell lung cancer with use of a next-generation sequencing cancer gene panel. JAMA Oncol 2019;5:696

29 Chae YK, Davis AA, Agte S, et al. Clinical implications of circulating tumor DNA tumor mutational burden (ctDNA TMB) in non-small cell lung cancer. Oncologist 2019;24:820-8.

30 Wang Z, Duan J, Wang G, et al. Allele Frequency-Adjusted BloodBased Tumor Mutational Burden as a Predictor of Overall Survival for Patients With NSCLC Treated With PD-(L)1 Inhibitors. J Thorac Oncol 2020;15:556-67.

31 FoundationOne_CDx_Label_Technical_Info. Available: https://info. foundationmedicine.com/hubfs/FMI Labels/FoundationOne_CDx Label_Technical_Info.pdf [Accessed 08 May 2021].
32 Tempus xT (V3) validation. Available: https://www.tempus.com/wpcontent/uploads/2020/02/xTv3-Validation_010920-1.pdf [Accessed 08 May 2021].

33 The Guardant 360 assay. Available: https://www.therapyselect. de/sites/default/files/downloads/guardant360/guardant360 specification-sheet_en.pdf [Accessed 08 May 2021].

34 Helman E, Artieri C, Vowles JV. Abstract 5603: analytical validation of a comprehensive 500-gene ctDNA panel designed for immunooncology and DNA damage research. Clin Res 2018.

35 Loree JM, Topham JT, Kennecke HF, et al. Tissue and plasma tumor mutation burden (TMB) as predictive biomarkers in the $\mathrm{CO} .26$ trial of durvalumab + tremelimumab $(\mathrm{D}+\mathrm{T})$ versus best supportive care (BSC) in metastatic colorectal cancer (mCRC). JCO 2021;39:61.

36 Fehrenbacher L, Spira A, Ballinger M, et al. Atezolizumab versus docetaxel for patients with previously treated non-small-cell lung cancer (poplar): a multicentre, open-label, phase 2 randomised controlled trial. Lancet 2016;387:1837-46.

37 Rittmeyer A, Barlesi F, Waterkamp D, et al. Atezolizumab versus docetaxel in patients with previously treated non-small-cell lung cancer (oak): a phase 3, open-label, multicentre randomised controlled trial. Lancet 2017;389:255-65.

38 Liu Z, Xie Z, Cai X, et al. A modified algorithm adjusting both high and minor allele frequency mutation to redefine blood-based tumor mutational burden (bTMB) for optimal prediction of clinical benefits from immune checkpoint inhibitor therapy. J Thorac Oncol 2020;15:e69-e72.

39 Abécassis J, Hamy A-S, Laurent C, et al. Assessing reliability of intra-tumor heterogeneity estimates from single sample whole exome sequencing data. PLoS One 2019;14:e0224143.

40 Roth A, Khattra J, Yap D, et al. PyClone: statistical inference of clonal population structure in cancer. Nat Methods 2014;11:396-8.

41 Ma F, Guan Y, Yi Z, et al. Assessing tumor heterogeneity using ctDNA to predict and monitor therapeutic response in metastatic breast cancer. Int J Cancer 2020;146:1359-68.

42 Morris LGT, Riaz N, Desrichard A, et al. Pan-Cancer analysis of intratumor heterogeneity as a prognostic determinant of survival. Oncotarget 2016;7:10051-63.

43 Dentro SC, Leshchiner I, Haase K, et al. Characterizing genetic intra-tumor heterogeneity across 2,658 human cancer genomes. Cell 2021:184:2239-2254.e39.

44 Miller CA, White BS, Dees ND, et al. SciClone: inferring clonal architecture and tracking the spatial and temporal patterns of tumor evolution. PLoS Comput Biol 2014;10:e1003665.

45 Hardiman KM, Ulintz PJ, Kuick RD, et al. Intra-Tumor genetic heterogeneity in rectal cancer. Lab Invest 2016;96:4-15.

46 Li X, Kumar S, Harmanci A, et al. Whole-Genome sequencing of phenotypically distinct inflammatory breast cancers reveals similar genomic alterations to non-inflammatory breast cancers. Genome Med 2021;13:70.

47 Liu Z, Xie Z, Zhao S, et al. Presence of allele frequency heterogeneity defined by ctDNA profiling predicts unfavorable overall survival of NSCLC. Trans/ Lung Cancer Res 2019;8:1045-50.

48 Mroz EA, Rocco JW. Math, a novel measure of intratumor genetic heterogeneity, is high in poor-outcome classes of head and neck squamous cell carcinoma. Oral Oncol 2013;49:211-5.

49 Mroz EA, Tward AD, Pickering CR, et al. High intratumor genetic heterogeneity is related to worse outcome in patients with head and neck squamous cell carcinoma. Cancer 2013;119:3034-42.

50 Mroz EA, Tward AD, Tward AM, Hammon RJ, et al. Intra-tumor genetic heterogeneity and mortality in head and neck cancer: analysis of data from the cancer genome atlas. PLoS Med 2015;12:e1001786

51 Rajput A, Bocklage T, Greenbaum A, et al. Mutant-allele tumor heterogeneity scores correlate with risk of metastases in colon cancer. Clin Colorectal Cancer 2017;16:e165-70.

52 Bettoni F, Masotti C, Corrêa BR. The effects of neoadjuvant chemoradiation in locally advanced rectal cancer-the impact in intratumoral heterogeneity. Frontiers in Oncology 2019;9.

53 Oh BY, Shin H-T, Yun JW, et al. Intratumor heterogeneity inferred from targeted deep sequencing as a prognostic indicator. Sci Rep 2019;9:4542.

54 Paschalis A, Sheehan B, Riisnaes R, et al. Prostate-specific membrane antigen heterogeneity and DNA repair defects in prostate cancer. Eur Urol 2019;76:469-78.

55 Chung YR, Kim HJ, Kim YA, et al. Diversity index as a novel prognostic factor in breast cancer. Oncotarget 2017;8:97114-26.

56 Choi J, Nam M, Fridland S, et al. 18 New method of assessing tumor heterogeneity utilizing both circulating tumor DNA and tissue DNA to predict the response to immunotherapy. $J$ Immunother Cancer 2020;8:A18. 